\title{
CORRECTION
}

View Article Online

View Journal I View Issue

D) Check for updates

Cite this: RSC Adv., 2021, 11, 34262

DOI: 10.1039/d1ra90161c

rsc.li/rsc-advances

\section{Correction: Unraveling variation on the profile aroma compounds of strong aroma type of Baijiu in different regions by molecular matrix analysis and olfactory analysis}

\author{
Jiaxin Hong, ${ }^{a}$ Junshan Wang, ${ }^{a}$ Chunsheng Zhang, ${ }^{b}$ Zhigang Zhao, ${ }^{b}$ Wenjing Tian, ${ }^{c}$ \\ Yashuai Wu, ${ }^{a}$ Hao Chen, ${ }^{a}$ Dongrui Zhao*a and Jinyuan Sun ${ }^{a}$ \\ Correction for 'Unraveling variation on the profile aroma compounds of strong aroma type of Baijiu in \\ different regions by molecular matrix analysis and olfactory analysis' by Jiaxin Hong et al., RSC Adv., \\ 2021, 11, 33511-33521. DOI: 10.1039/D1RA06073B.
}

The authors regret the omission of a funding acknowledgement in the original article. This acknowledgement is given below. Dongrui Zhao would like to acknowledge the National Natural Science Foundation of China (32001826).

The Royal Society of Chemistry apologises for these errors and any consequent inconvenience to authors and readers.

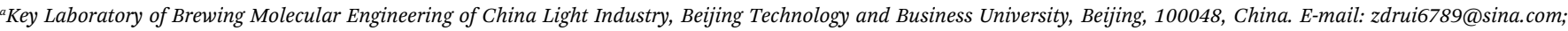
Fax: +86-10-68984890; Tel: +86-10-68988715

${ }^{b}$ Chengde Qianlongzui Distillery Company, Hebei, 100048, China

${ }^{c}$ Food and Biological Engineering, Beijing Vocational College of Agriculture, Beijing, 102442, China
} 